\title{
Caracterização química e energética para aproveitamento da madeira de costaneira e desbaste de cedro australiano
}

Lina Bufalino ${ }^{1}$, Thiago de Paula Protásio ${ }^{1}$, Allan Motta Couto ${ }^{1}$, Otávio Augusto Carvalho Nassur ${ }^{1}$, Vânia Aparecida de Sá1, Paulo Fernando Trugilho ${ }^{1}$, Lourival Marin Mendes ${ }^{1}$

1Universidade Federal de Lavras (UFLA), Departamento de Ciências Florestais, CP 3037, CEP 37200-000, Lavras, Minas Gerais, Brasil

*Autor correspondente:

linabufalino@yahoo.com.br

Termos para indexação:

Extrativos totais

Resíduos sólidos

Painéis reconstituídos

Poder calorífico

Toona ciliata

Index terms:

Total extractives

Solid wastes

Reconstituted panels

Higher heating value

Toona ciliata

Histórico do artigo:

Recebido em 11/01/2012

Aprovado em 29/05/2012

Publicado em 29/06/2012

doi: 10.4336/2012.pfb.32.70.13
Resumo - O objetivo desse trabalho foi quantificar e comparar as propriedades químicas e energéticas da madeira de cedro australiano Toona ciliata MJ Roem var. australis (FV Muell.) C. DC proveniente de desbaste e desdobro para produção de painéis reconstituídos e energia, além de verificar a eficiência da remoção de extrativos por tratamentos em água para viabilizar a produção de painéis de partículas. Os teores de lignina, holocelulose, extrativos totais, cinzas, carbono, hidrogênio, oxigênio, nitrogênio, enxofre e poder calorífico superior foram determinados. Dois tratamentos em água para remoção de extrativos foram realizados nos materiais: imersão em água fria durante 24 horas e em água fervente durante 2 horas. Foram encontrados menores teores de cinzas, holocelulose, hidrogênio e nitrogênio e maiores teores de lignina e extrativos totais nos resíduos de madeira proveniente de costaneiras de desdobro primário. Para as outras propriedades, os valores foram estatisticamente iguais. A madeira de cedro australiano apresenta elevado teor de extrativos, sendo o pré-tratamento em água necessário para a produção de determinados painéis de partículas. Os valores de poder calorífico dos materiais indicam potencial para a produção de energia.

\section{Chemical and energetic characterization for utilization of thinning and slab wood from Australian red cedar}

\begin{abstract}
This work aimed to quantify and compare chemical and energetic properties of Australian red cedar Toona ciliata MJ Roem var. australis (FV Muell.) C. DC wood from thinning and primary sawing for reconstituted panel and energy production; and also to verify the efficiency of extractive removal by water treatments, in order to improve wood quality for particleboard production. Lignin, holocellulose, extractives, ash, carbon, hydrogen, oxygen, nitrogen and sulfur contents and higher heating value were determined. Two water treatments for extractive removal were performed: immersion in cold water for 24 hours and immersion in boiling water for 2 hours. Lower values of ash, holocellulose, hydrogen and nitrogen contents and higher contents of lignin, total extractives, hydrogen and nitrogen contents were found for wood from primary sawing residues. For other properties, the values were significantly equal. Australian red cedar wood presents high extractive content, being water pre-treatment necessary for the production of some particleboards. Higher heating values of materials indicate potential for energy production.
\end{abstract}




\section{Introdução}

No Brasil, a área total de florestas plantadas com espécies dos gêneros Pinus e Eucalyptus totalizou 6.510 .693 ha no ano de 2010, sendo $73 \%$ correspondente à área de plantios de Eucalyptus e $27 \%$ de Pinus (Anuário..., 2011).

Os mais diversos setores madeireiros como moveleiro, celulose e papel, siderurgia e carvão vegetal, painéis de madeira industrializada e madeira processada mecanicamente são abastecidos predominantemente pela matéria-prima proveniente desses plantios florestais (Anuário..., 2011). No entanto, atualmente os produtores brasileiros têm investido em outras espécies que apresentam madeira com alto valor comercial. Essa prática é importante para garantir o suprimento de matéria prima e diversificar o mercado de produtos madeireiros do país.

Folhosas de madeira de alto valor e qualidade, que anteriormente só eram obtidas de florestas tropicais nativas, agora podem ser cultivadas com sistemas silviculturais complexos (Dordel, 2010). Nesse contexto, plantações da espécie Toona ciliata MJ Roem var. australis (FV Muell.) C. DC têm se destacado no Brasil. De acordo com o Centro de Inteligência em Florestas (2010), essa espécie encontra condições favoráveis para seu desenvolvimento nas regiões sudeste e sul da Bahia. Uma das vantagens do seu cultivo em diversos países da América Central e do Sul é que a mariposa da ponta (Hypsipyla grandela) de ocorrência nativa e que ataca os brotos terminais de espécies da família Meliaceae parece apresentar preferência pelas árvores dos gêneros Cedrela e Swietenia (Mayhew \& Newton, 1998).

A produção de madeira de Toona ciliata no Brasil vem sendo destinada para serrarias em função de sua elevada qualidade como grã reta, desenhos atrativos e alta resistência ao ataque de cupins (Ribeiro, 2011), além de ciclo produtivo relativamente curto, entre 15 e 20 anos de idade (Souza et al., 2009).

No entanto, é importante considerar que qualquer processamento mecânico da madeira gera resíduos em grande quantidade que na maioria das vezes são estocados, mas que poderiam ser utilizados industrialmente. Além do processamento primário e secundário da madeira, atividades silviculturais como o desbaste também resultam em oferta de matéria-prima sem qualidades atrativas para produção de madeira serrada. Dentre as possibilidades existentes para o aproveitamento de resíduos provenientes de desdobro e madeira de desbaste, as mais estudadas são a produção de painéis de madeira reconstituída (Suffian et al., 2010; Rowel, 2007; Setunge et al., 2009; Dacosta et al., 2005) e geração de energia de biomassa (Paula et al., 2011b; Protásio et al., 2011b; Khambalkar et al., 2008; Moghtaderi et al., 2006; Dermibas, 2004).

Entretanto, para promover o uso tecnológico correto e racional da madeira, a determinação e quantificação de suas propriedades são essenciais. A caracterização química apresenta implicações tecnológicas, tais como influência no processo de colagem da madeira e aproveitamento de resíduos para produção de energia. O poder calorífico e os teores de cinzas, lignina, holocelulose e de componentes elementares são propriedades diretamente relacionadas ao potencial energético da madeira (Paula et al., 2011a; Protásio et al., 2011a; Parikh et al., 2007; Moghtaderi et al., 2006; Sheng \& Azevedo, 2005; Mckendry, 2002; Schuster et al., 2000).

Para painéis de madeira reconstituída como Aglomerados/MDP e Oriented Strand Board (OSB), o teor de extrativos é a propriedade química mais importante, pois sua elevada concentração e migração, que ocorre durante o processo de secagem, podem bloquear o contato do adesivo com a madeira. De forma geral, espécies com altos teores de extrativos geram painéis cuja colagem é menos eficiente e de qualidade inferior em relação às com baixos teores. Além disso, extrativos em alta concentração podem dificultar a passagem da água e retardar a sua taxa de evaporação, o que aumenta o tempo de prensagem necessário (Frihart \& Hunt, 2010; Iwakiri, 2005; Mendes \& Albuquerque, 2000; Hsu, 1997). Yasar et al. (2010) sugerem o tratamento prévio de partículas de materiais lignocelulósicos em água ou solução alcalina para promover a remoção de parte dos extrativos e viabilizar a produção de painéis de partículas.

Os objetivos desse trabalho foram quantificar e comparar as propriedades químicas e energéticas da madeira de cedro australiano proveniente de desbaste e desdobro para produção de painéis de partículas e energia, além de verificar a eficiência da remoção de extrativos por tratamentos em água. 


\section{Material e métodos}

\section{Caracterização, preparo e amostragem do material}

As especificações da madeira de cedro australiano das duas fontes utilizadas para análises químicas e de poder calorífico estão apresentadas na Tabela 1 .

Tabela 1. Especificações dos materiais analisados

\begin{tabular}{ccccc}
\hline Madeira & Idade & $\begin{array}{c}\text { Material de } \\
\text { estudo }\end{array}$ & $\begin{array}{c}\text { Número } \\
\text { de } \\
\text { indivíduos }\end{array}$ & Procedência \\
\hline I & 4 anos & $\begin{array}{c}\text { Toras inteiras } \\
\text { de desbaste } \\
\text { pré-comercial }\end{array}$ & 5 & $\begin{array}{c}\text { Campo Belo, } \\
\text { MG - Brasil }\end{array}$ \\
II & 18 & $\begin{array}{c}\text { Costaneiras } \\
\text { geradas pelo } \\
\text { processamento } \\
\text { primário }\end{array}$ & 16 & $\begin{array}{c}\text { Marechal } \\
\text { Floriano, } \\
\text { anos - Brasil }\end{array}$ \\
\hline
\end{tabular}

As toras oriundas de desbaste foram transformadas em tábuas e costaneiras com o uso de uma serra de fita. Tanto as costaneiras oriundas do desdobro da madeira de 18 anos, quanto as tábuas e costaneiras provenientes da madeira de desbaste de 4 anos, foram transformadas em peças de dimensões $90 \mathrm{~mm}$ x $200 \mathrm{~mm}$ x $25 \mathrm{~mm}$, das quais foram obtidas partículas do tipo "strand" com dimensões nominais de 90 x $25 \times 0,7 \mathrm{~mm}$ por um picador de disco.

A amostragem foi executada nas partículas "strand" dos dois lotes pelo método do quarteamento de acordo com a Norma Brasileira Regulamentadora-NBR 1007 (Associação Brasileira de Normas Técnicas, 2004). Em seguida, as partículas de cada material foram divididas em três partes aproximadamente iguais, das quais uma não sofreu nenhum tipo de tratamento. As outras duas parcelas foram tratadas para retirada de parte dos extrativos, sendo uma submersa em água à temperatura ambiente por 24 horas ea outra emágua fervente por 2 horas. Após o tempo determinado, as partículas foram enxaguadas por um fluxo de água contínuo.

As partículas foram secas em condições ambiente e transformadas em serragem em moinho do tipo Willey. A classificação em função da granulometria foi executada por meio de um conjunto de peneiras. Os materiais foram separados para análise química de componentes moleculares (retidos entre 40 e 60 mesh), elementares (60 e 200 mesh) e poder calorífico superior (200 e 270 mesh). A serragem foi acondicionada em câmara climática a $20 \pm 3{ }^{\circ} \mathrm{C}$ e $60 \%$ de umidade relativa do ar até atingir a umidade de equilíbrio da madeira (12\%).

\section{Análises químicas das amostras}

As parcelas de serragem não tratadas da madeira de cedro australiano foram utilizadas para as análises dos teores de extrativos totais, lignina e cinzas. Para as parcelas tratadas em água foi empregada apenas a análise de extrativos totais. As normas utilizadas estão apresentadas na Tabela 2. O teor de holocelulose foi obtido pela diferença, conforme a Equação 1 (Neves et al., 2011):

Holocelulose $(\%)=100 \%-$ Extrativos $(\%)-$ Lignina $(\%)-$ Cinzas $(\%)(1)$

Tabela 2. Normas utilizadas para as análises químicas.

\begin{tabular}{cc}
\hline Análise & Norma utilizada \\
\hline Teor de extrativos totais & ABNT NBR 14853 (2010) \\
Teor de cinzas & ABNT NBR 13999 (2003) \\
Teor de lignina (klason) & ABNT NBR 7989 (2010) \\
\hline
\end{tabular}

Para a obtenção do poder calorífico superior, o material foi previamente seco em estufa a $103 \pm 2^{\circ} \mathrm{C}$ até massa constante. Uma bomba calorimétrica (IKA C-200) foi utilizada para as análises de poder calorífico superior de acordo com as recomendações da Norma Brasileira Regulamentadora 8633 (Associação Brasileira de Normas Técnicas, 1984).

Os teores dos componentes elementares carbono, hidrogênio, enxofre e nitrogênio foram determinados com um analisador elementar. $\mathrm{O}$ teor de oxigênio foi obtido pela Equação 2 (Bech et al., 2009):

$$
O(\%)=100 \%-C(\%)-H(\%)-N(\%)-S(\%)-\text { Cinzas }(\%)(2)
$$

\section{Análise estatística dos resultados}

De forma preliminar às análises de variância, foi realizado o teste de homogeneidade de variância (teste de Bartlett) a $5 \%$ de significância para todas as propriedades quantificadas.

A primeira análise foi realizada para a variável teor de extrativos totais para comparação entre três tratamentos: madeira não tratada; madeira tratada em água fria por 24 horas; e madeira tratada em água fervente por 2 horas. Os dados foram submetidos à análise de variância e comparados pelo teste de Tukey a 5\% de significância, considerando o delineamento inteiramente casualizado e três repetições com duas duplicatas.

As amostras de madeira de cedro australiano proveniente das duas fontes diferentes, costaneira e desbaste, correspondentes aos tratamentos, foram comparadas entre si, para as demais variáveis em estudo, pelo teste de $\mathrm{F}$ da análise de variância a $5 \%$ de significância. Para todas as variáveis foram utilizadas três repetições com duas duplicatas. 


\section{Resultados e discussão}

\section{Efeito do tratamento das partículas em água}

$\mathrm{O}$ teste de Bartlett indicou que a variável teor de extrativos totais apresenta homogeneidade de variância para madeira de desbaste ( $\mathrm{p}$ valor $=0,7493$ ) e de costaneira $(\mathrm{p}$ valor $=0,4557)$. Estes resultados validam os obtidos nas análises de variância. Na Tabela 3 estão apresentados os resultados das análises de variância realizadas para verificar a influência dos tratamentos da madeira de cedro australiano provenientes de desbaste e desdobro no teor de extrativos totais.

As partículas não tratadas, tratadas em água fria durante 24 horas ou tratada em água fervente durante 2 horas apresentaram teores de extrativos estatisticamente diferentes tanto para madeira proveniente de desbaste quanto de costaneiras. Os valores médios dos teores de extrativos totais e os resultados do teste de Tukey estão apresentados na Figura 1.

Tabela 3. Resumo das análises de variância para os teores de extrativos das partículas submetidas a diferentes tratamentos.

\begin{tabular}{cccc}
\hline \multirow{2}{*}{$\begin{array}{c}\text { Fonte de } \\
\text { Variação }\end{array}$} & $\begin{array}{c}\text { Graus de } \\
\text { Liberdade }\end{array}$ & \multicolumn{2}{c}{ Quadrado Médio } \\
\cline { 3 - 4 } Tratamento & 2 & $19,676^{*}$ & $35,192^{*}$ \\
Erro & 6 & 0,024 & 0,050 \\
\hline $\begin{array}{c}\text { Coeficiente de } \\
\text { Variação }(\%)\end{array}$ & - & 2,14 & 2,36 \\
\hline
\end{tabular}

*: Significativo a $5 \%$ de probabilidade, pelo teste Tuckey

A madeira de cedro australiano de ambas as fontes, sem tratamento, apresentou um alto teor de extrativos totais se comparada com as madeiras de florestas plantadas amplamente cultivadas e estudadas no Brasil. Para várias espécies do gênero Eucalyptus, os valores apresentados na literatura para teores de extrativos totais variam entre $1,5 \mathrm{e}$ 8\% (Mendes et al., 2011; Neves et al., 2011; Lima et al., 2007; Santos \& Sansígolo, 2007; Silvério et al., 2006; Trugilho et al.,1996, 2003, 2005), enquanto para as espécies de Pinus os estudos indicam valores de 3 a 6\% (Okino et al., 2007; Mendes et al., 2002; Shimoyama \& Wiecheteck, 1993; Tomazello Filho etal., 1985). O teor de extrativos totais relativamente alto pode ser observado em outras espécies da família Meliaceae, tais como Azadirachta indica A. Juss que apresentou 8,46\% com 4,2 anos de idade (Araújo et al., 2000) e Cedrela fissilis Vell., com 5,6\% de extrativos totais (Oliveira et al., 2005).
A diminuição significativa verificada para o teor de extrativos pode ser crucial do ponto de vista de produção de painéis de madeira reconstituída. Observações realizadas na Unidade Experimental de Produção de Painéis de Madeira (UFLA) já demonstraram a impossibilidade de produzir painéis OSB utilizando partículas obtidas de madeira de costaneira de cedro australiano sem prétratamento em água. O "estouro" dos painéis foi atribuído ao alto teor de extrativos. $\mathrm{O}$ mesmo não ocorreu para as partículas obtidas da madeira de desbaste.

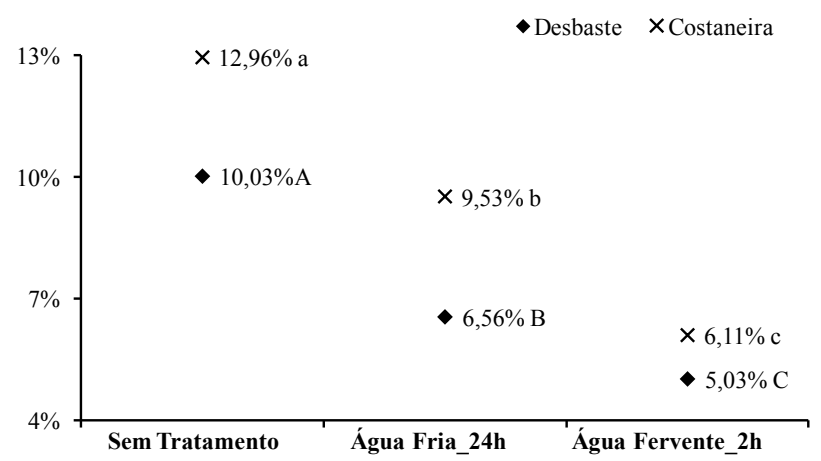

Figura 1. Teores de extrativos das partículas de cedro australiano submetidas aos diferentes tratamentos em água. As médias apresentaram diferenças significativas pelo teste Tuckey $(\mathrm{p}<0,05)$.

A imersão do material lignocelulósico em água tratase da opção mais viável técnica e economicamente quando comparada a tratamentos químicos como, por exemplo, a imersão em solução de hidróxido de sódio. A utilização de água fervente durante duas horas retira uma maior proporção de extrativos, mas demanda gasto energético. Entretanto, essa energia poderia ser obtida da cogeração por meio da queima dos resíduos gerados do processamento da madeira.

\section{Análise química molecular da madeira}

Foi verificada homogeneidade de variância entre os tratamentos (madeira de desbaste e costaneira) para teor de extrativos totais ( $\mathrm{p}$ valor $=0,7098$ ), teor de lignina $(\mathrm{p}$ valor $=0,4144)$, teor de cinzas $(\mathrm{p}$ valor $=0,6977) \mathrm{e}$ teor de holocelulose $(p=0,4094)$.

$\mathrm{Na}$ Tabela 4 estão apresentados os resumos das análises de variância realizadas para os teores dos componentes químicos moleculares de partículas de madeira de cedro australiano provenientes dos dois materiais em estudo. 
Tabela 4. Resumo das análises de variância dos teores dos componentes químicos moleculares para comparação entre materiais.

\begin{tabular}{|c|c|c|c|c|c|}
\hline \multirow{2}{*}{$\begin{array}{l}\text { Fonte de } \\
\text { Variação }\end{array}$} & \multirow{2}{*}{$\begin{array}{c}\text { Graus de } \\
\text { Liberdade }\end{array}$} & \multicolumn{4}{|c|}{ Quadrado Médio } \\
\hline & & Extrativos (\%) & Lignina (\%) & Cinzas (\%) & Holocelulose (\%) \\
\hline Material & 1 & $12,87^{*}$ & $11,98^{*}$ & $0,32 *$ & $41,87^{*}$ \\
\hline Erro & 4 & 0,05 & 0,41 & 0,00 & 0,20 \\
\hline CV $(\%)$ & - & 2,03 & 3,20 & 2,27 & 0,67 \\
\hline
\end{tabular}

*: significativo a $5 \%$ de probabilidade.

Houve diferença estatística significativa entre a madeira de cedro australiano proveniente de desbaste e costaneiras para todos os componentes químicos moleculares analisados. As composições moleculares da madeira de cedro australiano estão apresentadas na Figura 2.

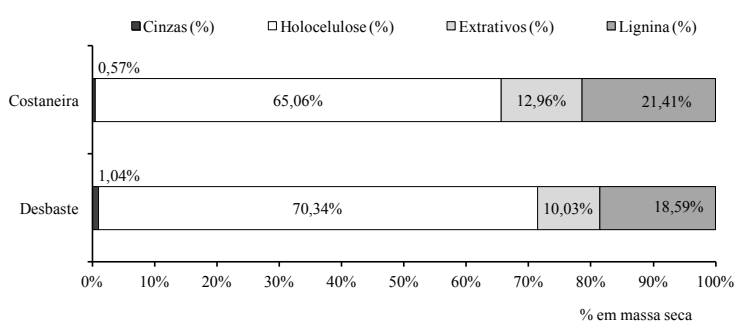

Figura 2. Composição química molecular das partículas de madeira de costaneira e de desbaste.

A madeira proveniente de costaneiras apresentou maior teor de extrativos totais em relação à madeira de desbaste. A presença de altos teores de extrativos é uma característica vantajosa para produção de energia devido ao elevado poder calorífico desses componentes (Guo et al., 2010; Telmo \& Lousada, 2011).

A holocelulose e a lignina são consideradas os principais componentes moleculares da biomassa. Para a produção de energia, maiores teores de lignina e menores teores de holocelulose são desejáveis, conforme observado para a madeira de cedro australiano obtida de costaneira.

Quanto maior o teor de lignina, maior será o poder calorífico dos combustíveis de biomassa, o que pode ser atribuído às ligações carbono-carbono entre as unidades monoméricas de fenil-propano presentes na lignina, que dificultam sua decomposição, e ao maior teor de carbono desse componente molecular da biomassa (Howard, 1973; Demirbas, 2001; Sharma et al., 2004). A holocelulose apresenta poder calorífico de $18,60 \mathrm{~kJ} \mathrm{~g}^{-1}$, enquanto que a lignina apresenta valores de poder calorífico entre 23,26 e 26,58 $\mathrm{kJ} \mathrm{g}^{-1}$ (Howard, 1973).
Os combustíveis da biomassa reagem em dois estágios diferentes durante a pirólise. No primeiro, a massa dos materiais lignocelulósicos decresce rapidamente em função da degradação térmica da celulose e hemiceluloses e, em seguida, durante o segundo estágio, a massa decresce de forma menos intensa com a degradação térmica da lignina, que é mais resistente (Gani \& Naruse, 2007).

$\mathrm{Na}$ literatura podem ser encontrados teores de lignina entre 27,6 e $30,5 \%$ para diversas espécies do gênero Eucalyptus aos quatro anos de idade (Trugilho, 2009) e 27,9 a 31,3\% para clones de Eucalyptus aos seis anos (Neves et al., 2011), os quais são consideravelmente superiores ao valor médio determinado nesse trabalho para madeira de cedro australiano proveniente de desbaste na mesma idade. Já para Pinus taeda e Eucalyptus grandis de 18 anos de idade, valores de $32 \mathrm{e}$ $27,8 \%$ de lignina foram determinados, respectivamente (Okino et al., 2007).

A obtenção de energia da biomassa normalmente é feita por meio de tecnologias termo-químicas, especialmente à combustão. O elevado teor de cinzas é desvantajoso porque diminui a transferência de calor no combustível e aumenta a corrosão dos equipamentos utilizados no processo (Tan \& Lagerkvist, 2011), além de diminuir o poder calorífico da biomassa (Paula et al., 2011a; Protásio et al., 2011a; Brand, 2010). Dessa forma, a madeira de cedro australiano proveniente de costaneira pode ser mais apta para a produção de energia em relação à madeira de desbaste, caso a eficiência técnica do processo seja considerada.

Os teores de cinzas de diversas espécies e clones de Eucalyptus variaram entre 0,15 e $0,83 \%$ nos trabalho realizados por Neves et al. (2011), Protásio et al. (2011a) e Ferreira et al. (1997), enquanto para espécies de Pinus foram relatados valores entre 0,15 e $0,25 \%$ (Mendes et al., 2002).

Maiores teores de lignina são desejáveis para a produção de painéis de madeira reconstituída. Esse 
componente apresenta estrutura muito condensada e aspecto irregular que propicia ao material alta rigidez, uma vez que atua como amortecedor das microfibrilas de celulose, limitando o movimento paralelo à grã e aumentando a resistência mecânica a forças externas (Sweet \& Winandy, 1999). Na literatura foi relatado que a inclusão de $50 \%$ da madeira de eucalipto, com maior teor de lignina, em painéis aglomerados de cedro australiano, resultou em um aumento da resistência à compressão (Bufalino et al., 2012).

Por se tratar de um adesivo natural, a lignina ainda pode contribuir para melhorar a adesão entre partículas dos painéis, resultando em melhor colagem e estabilidade dimensional (Joseleau et al., 2004; Khedari et al., 2004). Hashin et al. (2011) produziram painéis aglomerados sem a utilização de adesivo, promovendo colagem apenas pela decomposição da lignina. Isso é possível porque as reações de condensação da lignina durante o processo de prensagem contribuem para o mecanismo de colagem (Okuda et al., 2006). Dessa forma, os baixos teores desse componente encontrados para madeira de cedro australiano, especialmente proveniente de desbaste, constituem uma desvantagem para produção de painéis de partículas.

\section{Análise química elementar e poder calorífico superior da madeira}

Foi verificada homogeneidade de variância entre os tratamentos (madeira de desbaste e costaneira) para teor carbono ( $\mathrm{p}$ valor $=0,6042)$, teor de hidrogênio ( $\mathrm{p}$ valor $=0,9127$ ), teor de oxigênio ( $\mathrm{p}$ valor $=0,6392)$, teor de nitrogênio $(\mathrm{p}$ valor $=$ 0,6128 ), teor de enxofre ( $\mathrm{p}$ valor $=0,5755$ ) e poder calorífico superior ( $\mathrm{p}$ valor $=0,6722$ ).

$\mathrm{Na}$ Tabela 5 estão apresentados os resumos das análises de variância realizadas para verificar as diferenças estatísticas entre os teores dos componentes químicos elementares e o poder calorífico superior dos materiais. Diferenças estatísticas significativas foram observadas apenas para os teores de nitrogênio e hidrogênio. Os teores médios de carbono, oxigênio, hidrogênio e cinzas da madeira de cedro australiano para os dois materiais estão apresentados na Figura 3.

Os elementos químicos combustíveis principais são carbono e hidrogênio. O oxigênio não contribui para o aumento do poder calorífico do combustível (Paula et al., 2011a; Protásio et al., 2011a; Dermibas \& Demibas, 2009). Portanto, para a produção de energia é desejável que a madeira apresente elevados teores de carbono e hidrogênio e baixos teores de oxigênio e cinzas, em consequência às correlações respectivamente positivas e negativas existentes entre esses componentes e o poder calorífico (Paula et al., 2011a; Protásio et al., 2011a; Huang et al., 2009; Demirbas \& Demirbas, 2004). Dessa forma, o maior teor de hidrogênio encontrado para a madeira de desbaste é vantajoso. No entanto, esse material apresentou maior teor de cinzas.

A madeira de cedro australiano proveniente de desbaste e desdobro apresentou valores médios de teor de nitrogênio de 0,180 e $0,145 \%$, respectivamente. Para o teor de enxofre, o valor médio para os dois materiais foi de $0,05 \%$. O enxofre e o nitrogênio contribuem relativamente pouco na liberação de energia de um combustível, além de ocasionarem poluição do ambiente e formação de chuva ácida após sua liberação na atmosfera durante a combustão (Bilgen \& Kaygusuz, 2008). O nitrogênio está diretamente relacionado com a emissão de componentes $\mathrm{NO}_{\mathrm{x}}$, corrosão e deposição de cinzas (Dermibas, 2004). Já uma maior concentração de enxofre pode ocasionar a formação de $\mathrm{FeCl}_{2}$ e $\mathrm{ZnCl}_{2}$, componentes corrosivos nas caldeiras (Telmo et al., 2010).

Tabela 5. Resumo das análises de variância dos teores dos componentes químicos elementares para comparação entre materiais

\begin{tabular}{|c|c|c|c|c|c|c|c|}
\hline \multirow{2}{*}{$\begin{array}{l}\text { Fonte de } \\
\text { Variação }\end{array}$} & \multirow{2}{*}{$\begin{array}{c}\text { Grau de } \\
\text { Liberdade }\end{array}$} & \multicolumn{6}{|c|}{ Quadrado Médio } \\
\hline & & N (\%) & $\mathrm{C}(\%)$ & H (\%) & S (\%) & $\mathrm{O}(\%)$ & PCS (kcal kg-1) \\
\hline Material & 1 & $0,0018^{*}$ & $2,0172^{\text {ns }}$ & $0,1045^{*}$ & $0,0000^{\mathrm{ns}}$ & $3,1959^{\mathrm{ns}}$ & $3128,16^{\mathrm{ns}}$ \\
\hline Erro & 4 & 0,0002 & 0,7468 & 0,0098 & 0,0000 & 0,9277 & 1753,66 \\
\hline CV $(\%)$ & - & 7,84 & 1,75 & 1,48 & 18,05 & 2,20 & 0,90 \\
\hline
\end{tabular}

*: significativo a 5\% de probabilidade; ns: não significativo; PCS: poder calorífico superior. 


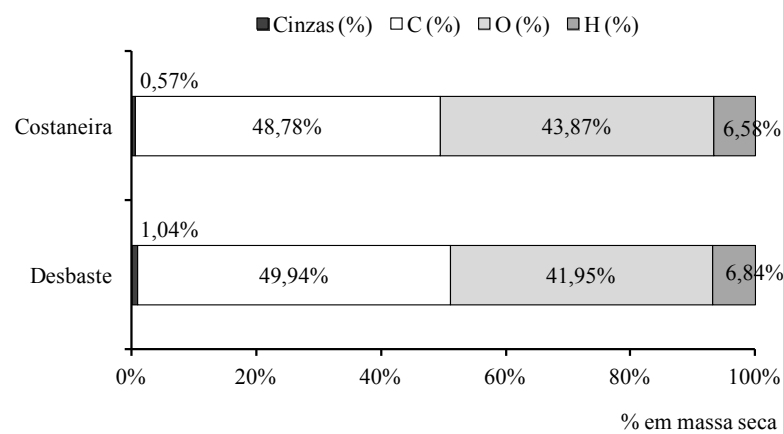

Figura 3. Teores dos componentes elementares e minerais da madeira de cedro australiano.

Assim como ocorre para a maioria das madeiras, as baixas concentrações de nitrogênio e enxofre encontradas para o cedro australiano não impedem a sua utilização energética, assemelhando-se a outros combustíveis de biomassa relatados na literatura (Neves et al., 2011; Paula et al., 2011a; Brand, 2010). Baixas quantidades desses componentes sugerem que os combustíveis são ambientalmente adequados para produção de energia via conversão termoquímica como, por exemplo, pirólise rápida (Pattiya, 2011).

Para o poder calorífico superior foram encontrados valores de 4.615,3 e 4.661,0 $\mathrm{kcal} \mathrm{kg}^{-1}$ para a madeira de cedro australiano proveniente de desbaste e desdobro, respectivamente. Telmo \& Lousada (2011) determinaram o poder calorífico superior da madeira de 17 espécies e encontraram valores entre 4.970,26 e 4.211,25 kcal kg-1. A madeira de cedro australiano proveniente de ambas as origens apresentou valores intermediários em relação aos observados pelos autores acima mencionados. No entanto, se comparadas a resíduos agrícolas, a madeira de cedro apresenta maior poder calorífico superior (Paula et al., 2011a; Protásio et al., 2011b).

O elevado poder calorífico superior encontrado para os dois materiais em estudo pode ser parcialmente atribuído aos seus altos teores de extrativos totais, visto que os teores de lignina observados foram baixos.

\section{Conclusões}

Foram encontrados menores teores de cinzas, holocelulose, hidrogênio e nitrogênio e maiores teores de lignina e extrativos totais nos resíduos de madeira proveniente de costaneiras de desdobro primário. Para as outras propriedades, os valores dos materiais foram estatisticamente iguais.
A madeira de cedro australiano proveniente de costaneiras apresentou elevado teor de extrativos, sendo o pré-tratamento em água para sua remoção necessário para a produção de painéis de madeira reconstituída.

A madeira de cedro australiano obtida por desbaste ou resíduos de desdobro primário apresenta potencial para produção de bioenergia em função de seu poder calorífico. Entretanto, a madeira de desbaste apresentou maior teor de cinzas.

\section{Referências}

ANUÁRIO Estatístico da ABRAF 2011: ano base 2010. Brasília, DF: ABRAF, 2011. 130 p.

ARAúJO, L. V. C. de; RODRIGUEZ, L. C. E.; PAES, J. B. Características físico-químicas e energéticas da madeira de nim indiano. Scientia Forestalis, Piracicaba, SP, n. 57, p. 153-159, 2000. ASSOCIAÇÃO BRASILEIRA DE NORMAS TÉCNICAS. NBR 10007: amostragem de resíduos sólidos. Rio de Janeiro, 2004. 25 p. ASSOCIAÇÃO BRASILEIRA DE NORMAS TÉCNICAS. NBR 13999: papel, cartão, pastas celulósicas e madeira: determinação do resíduo (cinza) após a incineração a $525^{\circ} \mathrm{C}$. Rio de Janeiro, 2003. 4 p. ASSOCIAÇÃO BRASILEIRA DE NORMAS TÉCNICAS. NBR 14853: madeira: determinação do material solúvel em etanol-tolueno e em diclorometano e em acetona. Rio de Janeiro, 2010. 3 p.

ASSOCIAÇÃO BRASILEIRA DE NORMAS TÉCNICAS. NBR 7989: pasta celulósica e madeira: determinação de lignina insolúvel em ácido. Rio de Janeiro, 2010. $6 \mathrm{p}$

ASSOCIAÇÃO BRASILEIRA DE NORMAS TÉCNICAS. NBR 8633: carvão vegetal: determinação do poder calorífico. Rio de Janeiro, 1984. 13 p.

BECH, N.; JENSEN, P. A.; DAM-JOHANSEN, K. Determining the elemental composition of fuels by bomb calorimetry and the inverse correlation of HHV with elemental composition. Biomass and Bioenergy, Amsterdam, v. 33, n. 3, p. 534-537, 2009.

BILGEN, S.; KAYGUSUZ, K. The calculation of the chemical exergies of coal-based fuels by using the higher heating values. Applied Energy, London, v. 85, n. 8, p. 776-785, 2008.

BRAND, M. A. Energia de biomassa florestal. Rio de Janeiro: Interciência, 2010. 131 p.

BUFALINO, L.; ABINO, V. C. S.; SÁ, V. A.; CORREA, A. A. R.; MENDES, L. M.; ALMEIDA, N. A. Particleboards made from Australian red cedar: processing variables and evaluation of mixed species. Journal of Tropical Forest Science, Kuala Lumpur, v. 24, n. 2, p. 162-172, 2012.

CENTRO DE INTELIGÊNCIA EM FLORESTAS (CIFlorestas).

Cedro australiano. Disponível em: $<\mathrm{http}$ ://www.ciflorestas.com.br/ texto.php? $\mathrm{p}=$ cedro_australiano $>$. Acesso em: 15 fev. 2011. 
DACOSTA, L. P. E.; HASELEIN, C. R.; SANTINI, E. J.; SCHNEIDER, P. R.; CALEGARI, L. Qualidade das chapas de partículas aglomeradas fabricadas com resíduos do processamento mecânico da madeira de Pinus elliottii Engelm. Ciência Florestal, Santa Maria, RS, v. 15, n. 3, p. 311-322, 2005.

DERMIBAS, A. Combustion characteristics of different biomass fuels. Progress in Energy and Combustion Science, Pittsburgh, v. 30, n. 2, p. 219-230, 2004.

DEMIRBAS, A. Relationships between lignin contents and heating values of biomass. Energy Conversion and Management, Oxford, v. 42, n. 2, p. 183-188, 2001.

DEMIRBAS, T.; DEMIRBAS, C. Fuel properties of wood species. Energy sources part A: recovery, utilization and environmental effects, London, v. 31, n. 16, p. 1464-1472, 2009.

DEMIRBAS, A.; DEMIRBAS, H. A. Estimating the calorific values of lignocellulosic fuels. Journal Energy, Exploration \& Exploitation, Georgetown, v. 20, n. 1, p. 105-111, 2004.

DORDEL, J.; SIMARD, S. W.; BAUHUS, J.; SEELY, B.; POZAS, L. J.; PRESCOTT, C.; HAMPEL, H. Trade-offs among establishment success, stem morphology and productivity of underplanted Toona ciliata: effects of nurse-species and thinning density. Forest Ecology and Management, Amsterdam, v. 259, n. 9, p. 1846-1855, 2010.

FERREIRA, G. W.; GONZAGA, J. V.; FOELKEL, C. E. B.; ASSIS, T. F.; RATNIEKS, E.; SILVA, M. C. M. Qualidade da celulose kraftantraquinona de Eucalyptus dunnii plantado em cinco espaçamentos em relação ao Eucalyptus grandis e Eucalyptus saligna. Ciência Florestal, Santa Maria, RS, v. 7, n. 1, p. 41-63, 1997.

FRIHART, C. R.; HUNT, C. G. Adhesives with wood materials: bond formation and performance. In: FOREST PRODUCTS LABORATORY. Wood handbook: wood as an engineering material. Washington, DC: USDA, 2010. p. 10/1-10/24.

GANI, A.; NARUSE, I. Effect of cellulose and lignin content on pyrolysis and combustion characteristics for several types of biomass. Renewable Energy, Oxford, v. 32, n. 4, p. 649-661, 2007.

GUO, X.; WANG, S.; WANG, K.; LIU, Q.; LUO, Z. Influence of extractives on mechanism of biomass pyrolysis. Journal of fuel Chemistry and Technology, v. 38, n. 1, p. 42-46, 2010.

HASHIM, R.; SAID, N.; LAMAMING, J.; BASKARAN, M.; SULAIMAN, O.; SATO, M.; HIZIROGLU, S.; SUGIMOTO, $\mathrm{T}$. Influence of press temperature on the properties of binderless particleboard made from oil palm trunk. Materials \& Design, Surrey, v. 32, n. 5, p. 2520-2525, 2011.

HOWARD, E. T. Heat of combustion of various Southern pine materials. Wood Science, v.5, n. 3, p.194- 197, 1973.

HSU, W. E. Wood quality requirements for panel products. In: INTERNATIONAL WOOD QUALITY WORKSHOP, 1., Oregon, 1997. Resumos... Oregon: CTIA/IUFRO, 1997.

HUANG, C.; HAN, L.; YANG, Z.; LIU, X. Ultimate analysis and heating value prediction of straw by near infrared spectroscopy. Waste Management, Oxford, v. 29, n. 6, p. 1793-1797, 2009.
IWAKIRI, S. Painéis de madeira. Curitiba: FUPEF, 2005. 247 p.

JOSELEAU, J. P.; IMAI, T.; KURODA, K.; RUEL, K. Detection in situ and characterization of lignin in the G-layer of tension wood fibres of Populus deltoids. Planta, Berlin, v. 219, n.2, p. 338-345, 2004. DOI 10.1007/s00425-004-1226-5

KHAMBALKAR, V. P.; KARALE, D. S.; GADGE, S. R.; DAHATONDE, S. B. Assessment of bioresources potential of rural village for self energy generation. BioResources, Raleigh, v. 3, n. 2, p. 566-575, 2008.

KHEDARI, J.; NANKONGNAB, N.; HIRUNLABH, J.; TEEKASAP, S. New low-cost insulation particleboards from mixture of durian peel and coconut coir. Building and Environment, Oxford, v. 39, n. 1, p. 59-65, 2004.

LIMA, C. K. P.; MORI, F. A.; MENDES, L. M.; CANEIRO, A. C. O. Características anatômicas e químicas da madeira de clones de Eucalyptus e sua influência na colagem. Cerne, Lavras, MG, v. 13, n. 2, p. 123-129, 2007.

MAYHEW, J. E.; NEWTON, A. C. The silviculture of mahogany. Edinburgh: CABI Publishing, 1998. 226 p.

MCKENDRY, P. Energy production from biomass (part 1): overview of biomass. Bioresource Technology, Kidlington, v. 83, n. 1, p. 37-46, 2002.

MENDES, L. M.; ALBUQUERQUE, C. E. C de. Aspectos técnicos e econômicos da indústria brasileira de chapas de fibras e partículas. Revista da Madeira, São Paulo, SP, n. 53, p. 14-22, 2000.

MENDES, L. M.; IWAKIRI, S.; MATOS, J. L. M.; KEINERT JR, S.; SALDANHA, L. K. Pinus spp. na produção de painéis de partículas orientadas (OSB). Ciência Florestal, Santa Maria, RS, v. 12, n. 2, p. $135-145,2002$.

MENDES, L. M.; LOSCHI, F. A. P.; PAULA, L. E. R.; MENDES, R. F.; GUIMARÃES JÚNIOR, J. B.; MORI, F. A. Potencial de utilização da madeira de clones de Eucalyptus urophylla na produção de painéis cimento-madeira. Cerne, Lavras, MG, v. 17, n. 1, p. 69-75, 2011.

MOGHTADERI, B.; SHENG, C.; WALL, T. F. An overview of the Australian biomass resources and utilization technologies. BioResources, Raleigh, v. 1, n. 1, p. 93-115, 2006.

NEVES, T. A.; PROTÁSIO, T. P.; COUTO, A. M.; TRUGILHO, P. F.; SILVA, V. O.; VIEIRA, C. M. M. Avaliação de clones de Eucalyptus em diferentes locais visando à produção de carvão vegetal. Pesquisa Florestal Brasileira, Colombo, PR, v. 31, n. 68, p. 319-330, 2011. DOI: 10.4336/2011.pfb.31.68.319

OKINO, E. Y. A.; ALVES, M. V. S.; TEIXEIRA, D. E.; SOUZA, M. R.; SANTANA, M. A. E. Biodegradação de chapas de partículas orientadas de pinus, eucalipto e cipreste expostas a quatro fungos apodrecedores. Scientia Forestalis, Piracicaba, SP, n. 74, p. 67-74, 2007.

OKUDA, N.; HORI, K.; SATO, M. Chemical changes of kenaf core binderless boards during hot pressing (II): effects on the binderless board properties. Journal of Wood Science, Tokyo, v. 52, n. 3, p. 249-254, 2006. 
OLIVEIRA, J. T. da S.; SOUZA, L. C. de; DELLA LUCIA, R. M.; SOUZA JUNIOR, W. P. Influência dos extrativos na resistência ao apodrecimento de seis espécies de madeira. Revista Árvore, Viçosa, MG, v. 29, n. 5, p. 819-826, 2005.

PARIKH, J.; CHANNIWALA, S. A.; GHOSAL, G. K. A correlation for calculating elemental composition from proximate analysis of biomass materials. Fuel, London, v. 86, n. 12-13, p. 1710-1719, 2007.

PATTIYA, A. Thermochemical characterization of agricultural wastes from thai cassava plantations. Energy Sources Part A: recovery utilization and environmental effects, London, v. 33, n. 8, p. 691-701, 2011.

PAULA, L. E. R.; TRUGILHO, P. F.; NAPOLI, A.; BIANCHI, M. L. Characterization of residues from plant biomass for use in energy generation. Cerne, Lavras, MG, v. 17, n. 2, p. 237-246, 2011 a.

PAULA, L. E. R.; TRUGILHO, P. F.; REZENDE, R. N.; ASSIS, C. O. Produção e avaliação de briquetes de resíduos lignocelulósicos. Pesquisa Florestal Brasileira, Colombo, PR, v. 31, n. 66, p. 103112, 2011b. DOI: 10.4336/2011.pfb.31.66.103

PROTÁSIO, T. P.; ALVES, I. C. N.; TRUGILHO, P. F.; SILVA, V. O.; BALIZA, A. E. R. Compactação de biomassa vegetal residual visando à produção de biocombustíveis sólidos. Pesquisa Florestal Brasileira, Colombo, PR, v. 31, n. 68, p. 273-283, 2011b. DOI: 10.4336/2011.pfb.31.68.273

PROTÁSIO, T. P.; BUFALINO, L.; TONOLI, G. H. D.; COUTO, A. M.; TRUGILHO, P. F.; GUIMARÃES JÚNIOR, M. Relação entre o poder calorífico superior e os componentes elementares e minerais da biomassa vegetal. Pesquisa Florestal Brasileira, Colombo, PR, v. 31, n. 66, p. 122 -133, 2011a. DOI: 10.4336/2011.pfb.31.66.113

RIBEIRO, M. X. Resistência de painéis aglomerados a cupins de madeira seca (Cryptotermes brevis). 2011. 92 f. Dissertação (Mestrado em Ciência e Tecnologia da Madeira) - Universidade Federal de Lavras, Lavras, MG.

ROWEL, R. M. Composite materials from forest biomass: a review of current practices, science, and technology. In: DIMITRIS S. A. (Ed.). Materials, chemicals, and energy from forest biomass. Carolina do Norte: ACS Publications, 2007. p 76-92.

SANTOS, S. R.; SANSÍGOLO, C. A. Influência da densidade básica da madeira de clones de Eucalyptus grandis x Eucalyptus urophylla na qualidade da polpa branqueada. Ciência Florestal, Santa Maria, RS, v. 17, n. 1, p. 53-63, 2007.

SCHUSTER, G.; LÖFFLER, G.; WEIGL, K.; HOFBAUER, H. Biomass steam gasification: an extensive parametric modeling study. Bioresource Technology, Kidlington, v. 77, n. 1, p. 71-79, 2000.

SHARMA, R. K.; WOOTEN, J. B.; BALIGA, V. L.; LIN, X.; CHAN, W. G.; HAJALIGOL, M. R. Characterization of chars from pyrolysis of lignin. Fuel, London, v. 83, n. 11-12, p. 1469-82, 2004.

SETUNGE, S.; WONG, K. K.; JOLLANDS, M. Economic and environmental benefits of using hardwood sawmill waste as raw material for particleboard production. Earth and Environmental Science, Edinburgh, v. 9, n. 5-6, p. 485-494, 2009.

SHENG, C.; AZEVEDO, J. L. T. Estimating the higher heating value of biomass fuels from basic analysis data. Biomass and Bioenergy, Amsterdam, v. 28, n. 5, p. 499-507, 2005.
SHIMOYAMA, V. R. S.; WIECHETECK, M. S. S. Características da madeira e da pasta termomecânica de Pinus patula var. tecunumanii para produção de papel imprensa. Série Técnica IPEF, Piracicaba, SP, v. 9, n. 27, p. 63-80, 1993.

SILVÉRIO, F. O.; BARBOSA, L. C. A.; GOMIDE, J. L.; REIS, F. P.; PILÓ-VELOSO, D. Metodologia de extração e determinação do teor de extrativos em madeiras de eucalipto. Revista Árvore, Viçosa, MG, v. 30, n. 6, p. 1009-1016, 2006.

SOUZA, J. C. A. V.; BARROSO, D. G.; CARNEIRO, J. G. A.; TEIXEIRA, S. L.; BALBINOT, E. Propagação vegetativa de cedro australiano (Toona ciliata M. Roemer) por miniestaquia. Revista Árvore, Viçosa, MG, v. 33, n. 2, p. 205-213, 2009.

SUFFIAN, M.; ORMONDROYD, G. A.; HALE, M. D. Comparisons of particleboard produced from Acacia hybrid and a UK commercial particleboard furnish from recycled wood. Journal of Tropical Forest Science, Kepong, v. 22, n. 3, p. 227-236, 2010.

SWEET, M. S.; WINANDY, J. E. The influence of degree of polymerization (DP) of cellulose and hemicellulose on the strength loss of fire-retardant-treated wood. Holzforschung, Berlin, v. 53, n. 3, p. 311-317, 1999.

TAN, Z.; LAGERLVIST, A. Phosphorous recovery from the biomass ash: a review. Renewable and Sustainable Energy Reviews, v. 15, n. 8, p. 3588-3602, 2011.

TELMO, C.; LOUSADA, J. Heating values of wood pellets from different species. Biomass and Bioenergy, Oxford, v. 35, n. 7, p. 2634-2639, 2011.

TELMO, C.; LOUSADA, J.; MOREIRA, N. Proximate analysis, backwards stepwise regression between gross calorific value, ultimate and chemical analysis of wood. Bioresource Technology, Essex, v. 101, n.11, p. 3808-3815, 2010.

TOMAZELLO FILHO, M.; LUIZ E. C.; BARRICHELO, L. E. C.; COSTA, J. C. Análise da madeira de compressão em Pinus oocarpa e Pinus strobus var. chiapensis. Composição química. IPEF, Piracicaba, SP, n. 31, p. 69-73, 1985.

TRUGILHO, P. F. Densidade básica e estimativa de massa seca e de lignina na madeira em espécies de Eucalyptus. Ciência e Agrotecnologia, Lavras, MG, v. 33, n. 5, p. 1228-1239, 2009.

TRUGILHO, P. F.; BIANCHI, M. L.; GOMIDE, J. L.; LIMA, J. T.; MENDES, L. M.; MORI, F. A.; GOMES, D. F. F. Clones de Eucalyptus versus a produção de polpa celulósica. Ciência Florestal, Santa Maria, RS, v. 15, n. 2, p. 145-155, 2005.

TRUGILHO, P. F.; LIMA, J. T.; MORI, F. A. Correlação canônica das características químicas e físicas da madeira de clones de Eucalyptus grandis e Eucalyptus saligna. Cerne, Lavras, MG, v. 9, n. 1, p. 66-80, 2003.

TRUGILHO, P. F.; LIMA, J. T.; MENDES, L. M. Influência da idade nas características físico-químicas e anatômicas da madeira de Eucalyptus saligna. Cerne, Lavras, MG, v. 2, n. 1, p. 94-11, 1996.

YASAR, S.; GUNTEKIN, E.; CENGIZ, M.; TANRIVERDI, H. The correlation of chemical characteristics and UF resin ratios to physical and mechanical properties of particleboard manufactured from vine prunings. Scientific Research and Essays, Nairobi, v. 5, n. 8 , p. $737-741,2010$. 
